\title{
ASSOCIAÇÃO ENTRE IDADE DA MENARCA E ESTATURA DEFINITIVA NO ESTUDO PRÓ-SAÚDE
}

\author{
Marcos Junqueira do Lago, Eduardo Faerstein, Rosely Sichieri, Claudia S. Lopes, Guilherme L. Werneck* \\ Trabalho realizado no Departamento de Epidemiologia do Instituto de Medicina Social da Universidade do Estado do Rio de Janeiro (Ueri), Rio de \\ Janeiro, RJ.
}

\author{
*Correspondência: \\ Instituto de Medicina Social/ \\ IMS, Ueri \\ Rua São Francisco Xavier \\ $524,7^{\circ}$ andar, Bloco D \\ Maracanã, Rio de Janeiro, RJ \\ Cep: $20559-900$ \\ Tel: (21) 2587-7303 \\ Fax: (21) 2264-1142 \\ gwerneck@nesc.ufri.br
}

\begin{abstract}
RESUMO
Oвjetıvo. Avaliar a associação entre idade da menarca e estatura.

Métodos. Por meio de questionário autopreenchível, foram obtidos dados acerca da data de nascimento, idade à primeira menstruação e grau de escolaridade do pai e da mãe para 1940 funcionárias técnico-administrativas de uma universidade localizada no Rio de Janeiro (Estudo Pró-Saúde). A estatura foi aferida duas vezes, em centímetros, por antropometristas treinados. A associação entre a idade da menarca e a estatura foi analisada por meio de regressão linear, tendo a estatura como variável dependente.
\end{abstract}

Resultados. Após o ajuste para nível educacional do pai e da mãe, observou-se que cada ano adicional na idade da menarca esteve associado com incremento de $0,91 \mathrm{~cm}, 0,44 \mathrm{~cm}$ e $0,40 \mathrm{~cm}$ na estatura de mulheres nas faixas etárias de 22 a 30 anos, 3 I a 40 anos e 4 I a 50 anos, respectivamente. Para as mulheres entre 5 I e 60 anos de idade, contudo, para cada ano a mais na idade da menarca houve uma diminuição na estatura de $0,64 \mathrm{~cm}$. Analisando-se apenas mulheres de até 50 anos de idade, não foi possível evidenciar interação entre faixa etária e idade da menarca, mas um efeito negativo de confusão exercido pela idade das participantes e pelo nível educacional de seus pais.

Conclusão. Os resultados deste estudo sugerem que, para mulheres de gerações mais recentes, a idade da menarca está diretamente associada à estatura definitiva. As condições socioeconômicas durante a infância e o efeito de geração podem ser importantes fatores de confusão e merecem ser considerados em análises futuras.

UnItERMOS: Estatura. Menarca. Nível socioeconômico.

\section{INTRODUÇÃO}

Tendências seculares de antecipação da idade da menarca e de aumento da estatura têm sido observadas em sociedades com diferentes graus de desenvolvimento econômico e social',2. No Brasil, entre 1975 e 1989, houve um aumento de $3,1 \mathrm{~cm}$ na estatura de homens aos 18 anos de idade ${ }^{3}$. Observou-se, ainda, uma antecipação da idade da menarca de 2,6 e 2,4 meses por década para mulheres nascidas, respectivamente, entre 1920 e $1979^{4}$ e 1932 e $1977^{5}$. A influência de fatores culturais, étnicos, genéticos, intra-uterinos e perinatais na idade da menarca e na estatura final tem sido freqüentemente estudada'. Alguns estudos destacam, também, o impacto potencial das condições socioeconômicas durante a infância e da coorte de nascimento na estatura e idade da primeira menstruaçãã 1,2,6.

Em grande parte dos estudos ${ }^{7-10}$, embora não em todos $^{11-14}$, observou-se uma associação direta entre a idade da menarca e a estatura final, o que poderia ser explicado pelo fato do momento da primeira menstruação relacionar-se fortemente ao estirão de crescimento ${ }^{6}$. Entretanto, alguns estudos descrevem uma relação não linear entre essas variáveis, em que maior estatura na vida adulta seria observada entre mulheres com idade da primeira menstruação próxima à média observada para a populaçãa estudada ${ }^{15,16}$.
No Brasil, Crespin " não observou uma associação evidente entre idade da menarca e estatura aos 16 anos em 120 mulheres pertencentes às classes média ou alta. Lobato \& Silveira' ${ }^{13}$, no Rio Grande do Sul, também não observaram associação entre idade da menarca e estatura definitiva em um grupo de 1005 mulheres de classe média. No entanto, em ambos os estudos, os métodos analíticos utilizados não foram bem explicitados, nem co-variáveis relevantes consideradas.

No presente artigo, descreve-se a relação entre idade da menarca e estatura final em uma população de funcionárias públicas brasileiras, levando-se em consideração os efeitos da geração e das condições socioeconômicas vividas na infância.

\section{Métodos}

O Pró-Saúde constitui-se de um estudo de coorte de funcionários técnico-administrativos de uma universidade localizada no Estado do Rio de Janeiro. Em relação a um total de 4448 funcionários elegíveis, 4 I 8 (9,4\%) não participaram da fase I do estudo em razão de recusas ou não localização. Entre 4030 funcionários participantes, 2240 $(55,6 \%)$ eram do sexo feminino. Para a análise do presente estudo, foram consideradas 1940 funcionárias (86,6\% do total de mulheres), após exclusão das participantes com idade superior a 60 anos (42 mulheres, 1,9\%) ou com dados faltantes para uma ou mais variáveis 
Tabela I - Estatura final e idade da menarca (médias e desvios padrão) segundo faixa etária e grau de escolaridade do pai e da mãe das participantes. Estudo Pró-Saúde, 1999

\begin{tabular}{|c|c|c|c|c|c|}
\hline \multirow[b]{2}{*}{ Faixa etária (anos) } & \multirow[b]{2}{*}{$\mathbf{N}$} & \multicolumn{2}{|c|}{ Estatura (metros) } & \multicolumn{2}{|c|}{ Idade da menarca (anos) } \\
\hline & & Média(DP) & $p^{*}$ & Média(DP) & $p^{*}$ \\
\hline $22-30$ & $\overline{239}$ & $\overline{1,62(0,57)}$ & & $\overline{12,15(1,39)}$ & \\
\hline $31-40$ & 834 & $1,61(0,61)$ & & $12,16(1,59)$ & \\
\hline $41-50$ & 666 & $1,59(0,63)$ & $<0,01$ & $12,35(1,66)$ & $<0,0$ \\
\hline $51-60$ & 201 & $1,58(0,67)$ & & $|2,6|(1,81)$ & \\
\hline \multicolumn{6}{|c|}{ Grau de escolaridade do pai } \\
\hline Não freqüentou a escola & 164 & $1,59(0,61)$ & & $12,66(1,59)$ & \\
\hline $1^{\circ}$ grau incompleto & 664 & $1,59(0,61)$ & & $12,37(1,68)$ & \\
\hline I’ grau completo $^{\circ}$ & 384 & $1,60(0,61)$ & $<0,01$ & $12,32(1,59)$ & $<0,0$ \\
\hline $2^{\circ}$ grau incompleto & $|0|$ & $1,60(0,62)$ & & $12,30(1,53)$ & \\
\hline $2^{\circ}$ grau completo & 326 & $\mid, 61(0,65)$ & & $12,02(1,57)$ & \\
\hline Universitário incompleto & 44 & $1,62(0,69)$ & & $12,27(1,74)$ & \\
\hline Universitário completo & 257 & $1,62(0,63)$ & & $11,99(1,53)$ & \\
\hline \multicolumn{6}{|c|}{ Grau de escolaridade da mãe } \\
\hline Não freqüentou a escola & 238 & $1,59(0,60)$ & & $12,60(1,67)$ & \\
\hline $1^{\circ}$ grau incompleto & 765 & $1,60(0,64)$ & & $12,37(1,67)$ & \\
\hline I grau completo & 390 & $1,60(0,60)$ & $<0,01$ & $12,20(1,63)$ & $<0,0$ \\
\hline $2^{\circ}$ grau incompleto & 99 & $1,59(0,67)$ & & $12,22(1,61)$ & \\
\hline $2^{\circ}$ grau completo & 294 & $1,62(0,62)$ & & $12,00(1,48)$ & \\
\hline Universitário incompleto & 34 & $1,62(0,59)$ & & $12,24(1,33)$ & \\
\hline Universitário completo & 120 & $1,62(0,53)$ & & $11,99(1,43)$ & \\
\hline
\end{tabular}

* p: valor do teste para tendência linear

incluídas nas análises (258, 11,5\%). A exclusão de mulheres acima de 60 anos de idade baseou-se na possibilidade de alteração da estatura devido à diminuição dos espaços intervertebrais decorrente da idade ${ }^{17}$

Informações sobre características de interesse foram obtidas a partir de um questionário multidimensional autopreenchível, semiestruturado ${ }^{18}$. A coleta de dados foi realizada no segundo semestre de 1999, durante o horário do expediente, com apoio de aplicadores treinados. A idade da menarca foi obtida pela pergunta "Com que idade você ficou menstruada pela primeira vez?", respondida em anos completos. Os graus de escolaridade do pai e da mãe foram utilizados como indicadores do nível socioeconômico na infância. A estatura foi aferida por duas vezes em centímetros, por meio de medição com fita métrica não elástica, que foi fixada com auxílio de prumo, em superfície vertical, sem rodapé. As medidas foram verificadas com um esquadro de madeira repousado sobre a cabeça, por antropometristas previamente treinados. Para fins de análise, foi utilizada a média aritmética das duas medidas.

Estudo piloto e análises de confiabilidade teste-reteste com intervalo de duas semanas revelaram boa qualidade do processo de obtenção dos dados. Em relação às características estudadas, utilizando-se a estatística Kappa com pesos quadráticos (k) para variáveis ordinais e o coeficiente de correlação intraclasse (ICC) para variáveis contínuas, foi observada confiabilidade de $k=0,93$ (escolaridade do pai); $k=0,99$ (escolaridade da mãe) e ICC $=0,89$ (idade da menarca).
A associação entre estatura (variável dependente) e idade da menarca (variável independente) foi investigada por meio de modelos de regressão linear, tendo como co-variáveis a idade atual das participantes e a escolaridade do pai e da mãe. As variáveis escolaridade do pai e a da mãe foram inseridas nos modelos de análise de forma ordinal $\left(<1^{\circ}\right.$ grau; $\geq 1^{\circ}$ grau completo a $<2^{\circ}$ grau e $\geq 2^{\circ}$ grau completo). Foram testadas interações entre a idade da menarca e as co-variáveis, comparando-se a verossimilhança dos modelos com e sem o termo de interação. Para a entrada dos dados, realizada com dupla digitação independente, utilizou-se o programa EPI-Info 6.0, e para a análise dos dados o programa Stata $6^{\text {TM }}$.

Os protocolos referentes à pesquisa foram aprovados pelos Comitês de Ética da universidade e do hospital universitário. Foram esclarecidos o caráter voluntário da participação e a natureza confidencial das informações, antes da leitura e assinatura de termo de consentimento.

\section{Resultados}

A Tabela I apresenta a distribuição da estatura final e da idade da menarca segundo faixas etárias, e escolaridade do pai e da mãe das participantes. Observou-se que a estatura média das participantes variou de forma inversa com a faixa etária e de forma direta com a escolaridade de seus pais. A idade média da menarca, por sua vez, variou diretamente com a idade das participantes e inversamente com a escolaridade de seus pais. Essas tendências lineares revelaram-se estatisticamente significativas $(p<0,01)$. 
Tabela 2 - Associações entre idade da menarca* (variável independente) e estatura final** (variável dependente) para diferentes faixas etárias das participantes. Estudo Pró-Saúde, 1999

\begin{tabular}{|c|c|c|c|c|c|}
\hline \multirow[b]{2}{*}{ Faixa etária (anos) } & \multirow[b]{2}{*}{$\mathrm{n}$} & \multicolumn{2}{|c|}{ Modelos sem ajuste para escolaridade dos pais } & \multicolumn{2}{|c|}{ Modelos ajustados para escolaridade dos pai } \\
\hline & & $\beta^{* * *}$ & IC 95\%"*** & $\beta^{* * *}$ & IC 95\%"*** \\
\hline $\begin{array}{l}22-30 \\
31-40 \\
41-50 \\
51-60\end{array}$ & $\begin{array}{l}239 \\
834 \\
666 \\
201\end{array}$ & $\begin{array}{l}0,90 \\
0,38 \\
0,30 \\
-0,71\end{array}$ & $\begin{array}{r}0,39-1,41 \\
0,12-0,64 \\
0,02-0,59 \\
-1,22--0,20\end{array}$ & $\begin{array}{l}0,91 \\
0,44 \\
0,40 \\
-0,64\end{array}$ & $\begin{array}{rr}0,41 & -1,42 \\
0,18 & -0,70 \\
0,12 & -0,68 \\
-1,16 & --1,32\end{array}$ \\
\hline
\end{tabular}

* Em anos; *** Em centímetros; **** Coeficiente de regressão linear; ***** Intervalo de 95\% de confiança

Tabela 3 - Associação entre idade da menarca* (variável independente) e estatura final** (variável dependente) entre mulheres na faixa etária de idade 22 a 50 anos. Estudo Pró-Saúde, 1999

\begin{tabular}{|c|c|c|}
\hline Variáveis independentes no modelo & $\beta^{* * *}$ & IC $95 \% \%^{* * * *}$ \\
\hline Modelo I: idade da menarca & $\overline{0,34}$ & $0,16-0,52$ \\
\hline Modelo 2: idade da menarca + idade & 0,42 & $0,25-0,60$ \\
\hline
\end{tabular}

* Em anos; * Em centímetros; **** Coeficiente de regressão linear; ***** Intervalo de 95\% de confiança

Gráfico I - Predição linear da altura segundo idade da menarca para diferentes faixas etárias. Estudo Pró-Saúde, 1999

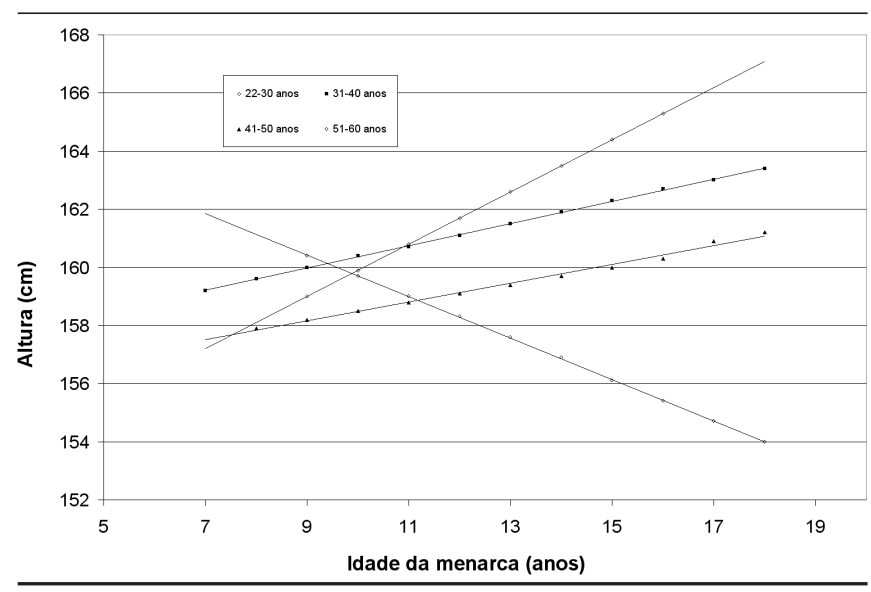

$\mathrm{Na}$ Tabela 2, observa-se uma associação direta entre idade da menarca e estatura para mulheres até 50 anos de idade. Controlando para o efeito da escolaridade do pai e da mãe, observou-se um aumento na estatura de 0,91 cm, 0,44 cm e 0,40 cm entre as funcionárias de 22 a 30 anos, 3 I a 40 anos e 4 I a 50 anos de idade, respectivamente, para cada ano a mais na idade da menarca. Em contraste, entre as funcionárias na faixa etária de 5 I a 60 anos, para cada ano a mais na idade da menarca, observou-se uma diminuição na estatura de 0,64 cm. Este efeito modificador exercido pela idade atual na associação entre idade da menarca e altura pode ser melhor visualizado no Gráfico I.

Excluindo-se da análise as mulheres de 5 I a 60 anos de idade, não mais foi possível identificar uma interação significativa entre idade da menarca e faixa etária. A Tabela 3 mostra diferentes modelos para avaliação da associação entre idade da menarca e estatura definitiva, apenas para as participantes com idade até 50 anos. O modelo I representa a associação simples (não ajustada por outras variáveis) entre idade da menarca e estatura definitiva. $O$ modelo 2 avalia a mesma associação, porém controlando para o efeito da idade das participantes. Já o modelo 3 inclui também a escolaridade dos pais como variáveis de ajuste. Evidencia-se um forte efeito negativo de confusão exercido pela idade das participantes e pelo nível educacional de seus pais, de forma que, no modelo ajustado por estas variáveis (modelo 3), o acréscimo na estatura é cerca $0,47 \mathrm{~cm}$ para cada ano a mais na idade da menarca, um incremento de cerca de $38 \%$ quando comparado com o modelo não ajustado (modelo I).

\section{Discussão}

Os trabalhos publicados sobre o tema abordado neste artigo têm, em sua maioria, relatado uma associação direta entre idade da menarca e estatura final ${ }^{7-10}$. Também neste estudo observou-se que uma menarca mais tardia está associada a maior altura definitiva para as mulheres com até 50 anos de idade, com ganhos estaturais da ordem de 0,4 a 0,9 cm para cada ano adicional na idade da menarca, na dependência da faixa etária considerada.

Para estas mulheres, uma associação direta mais forte entre idade da menarca e altura definitiva pode ser observada após o controle pela idade e pelo grau de educação do pai e da mãe, com um incremento de cerca de $38 \%$ na estatura final quando se compara o modelo ajustado ao não ajustado. É plausível que o nível socioeconômico na infância possa atuar como um fator de confusão na relação entre idade da menarca e altura. Por um lado, uma infância vivida em condições desfavoráveis poderia contribuir para o retardamento da idade da menarca na medida em que a criança levaria mais tempo até adquirir o estado nutricional necessário para o seu pleno desenvolvimento 
puberal. Por outro, estas mesmas condições poderiam levar a um subaproveitamento do potencial genético para altura levando, por sua vez, a uma menor estatura definitiva. Dessa forma, condições desfavoráveis ao crescimento e desenvolvimento durante o período pré-puberal estariam relacionadas a um retardo para a idade da menarca e a uma menor estatura final. No entanto, parte desta associação poderia ser explicada pelas estaturas do pai e da mãe, reconhecidos preditores da estatura final da prole ${ }^{19,20}$, mas estas informações não estavam disponíveis, o que configura uma importante limitação deste estudo.

Para as mulheres entre 5I e 60 anos de idade, contudo, observou-se um efeito inverso da idade da menarca, com uma diminuiçã̃o de 0,64 cm na estatura para cada ano a mais na idade da primeira menstruação. Este papel modificador exercido pela geração - captada neste estudo via idade das participantes - no efeito da idade da menarca é intrigante e não parece ter sido ainda descrito. É possível que a relação inversa observada para as participantes acima de 50 anos de idade seja reflexo de um efeito de coorte ou de período. Estas mulheres, nascidas entre os anos de 1938 e 1948, viveram suas infâncias durante e logo após a Segunda Guerra Mundial. É plausível que, para esta coorte de nascimento ou mesmo para este período, as mulheres com menarca mais precoce tenham sido justamente aquelas que tiveram melhores condições de vida durante a infância, tendo por este último aspecto conseguido alcançar uma melhor estatura definitiva. Nesse sentido, aquelas mulheres que apresentaram idade da menarca mais tardia seriam as que viveram sob condições materiais de vida mais precárias, sujeitas a processos infecciosos e nutricionais deletérios que podem ter afetado seu potencial para alcançar uma maior altura definitiva. É possível que, no caso específico dessa geração, o grau de educação dos pais não seja um indicador adequado da situação socioeconômica vivida na infância, não permitindo, portanto, um apropriado controle, ao contrário do que pode ser feito para as gerações mais recentes. Para estas, a educação paterna e materna indicaria adequadamente as condições materiais de vida durante a infância, de forma que, após o ajuste para esta variável, o efeito positivo da idade da menarca mais tardia na estatura final pode ser detectado de forma não subestimada.

\section{Conclusão}

A idade da menarca não pode ser considerada apenas como um evento que exerce isoladamente influência sobre a estatura final. O momento da primeira menstruação é um marco bem definido de uma trajetória que se inicia na vida intra-uterina, e que sofre inúmeras influências durante a infância. Considerando que, no momento da primeira menstruação, a maioria das mulheres já alcançou algo em torno de $95 \%$ da sua estatura final ${ }^{6}$, uma menarca mais tardia - e, em decorrência, tempo e eficácia maiores para o crescimento - tenderia a estar associada a uma maior estatura final.

Os achados do presente estudo sustentam a hipótese de que, pelo menos para as gerações mais jovens, a idade da menarca está diretamente associada à estatura final. Nossos resultados também sugerem que estudos sobre esta associação devem considerar o efeito da geração e das condições materiais de vida durante a infância.

\section{Conflito de interesse: não há}

\section{SUMMARY}

\section{Relation between age at menarche and final height of women in the Pró-Saúde Study}

OBIECTIVE. To evaluate the relation between age at menarche and final height.

METHODS. Complete data were obtained for 1,940 participants of a cohort study (Pró-Saúde Study) of civil servants at a university in Rio de Janeiro. A self-administered questionnaire was used to obtain the date of birth, age of the first menstruation and parents' educational level. Height in centimeters was measured twice by trained anthropometrists. Data were analyzed using linear regression models with final height as the dependent variable.

RESULTS. After adjusting for parents' educational level, it was observed that for each additional year in the age at menarche there was an increase in the final height of $0.91 \mathrm{~cm}$ for women aged 22 to 30 years, $0.44 \mathrm{~cm}$ for women aged 31 to 40 years and $0.40 \mathrm{~cm}$ for those between $4 I$ and 50 years of age. In contrast, for women of 51 to 60 years of age, each additional year in the age at menarche was associated with a decrease of $0.64 \mathrm{~cm}$ in the final height. Focusing only on women between 22 and 50 years of age, no significant interaction was identified between age and age at menarche, however a strong negative confounding effect exerted by participants' age and by their parents' educational level was identified.

CONCLUSION. Results ofthis study suggest that, foryoungergenerations, a later age at menarche is directly associated with final height. Childhood socioeconomic conditions and generation effects can be important confounding factors and should be considered in analyses of this relationship. [Rev Assoc Med Bras 2007; 53(I): 20-4]

KEY wORDS: Final height. Menarche. Socioeconomic level.

\section{REFERÊNCIAS}

I. Hauspie RC, Vercauteren M, Susanne C. Secular changes in growth and maturation: an update. Acta Paediatric Suppl. 1997;423:20-7.

2. Kac G. Tendência secular em estatura: uma revisão da literatura. Cad Saúde Pública. 1999; | 5: 45 I-6I.

3. Ministério da Saúde. Instituto Nacional de Alimentação e Nutrição. Pesquisa nacional sobre saúde e nutrição: perfil de crescimento da população brasileira de 0 a 25 anos. Brasília: INAN; 1990.

4. Kac G, Coel ASC, Velasquez-Melendez G. Secular trend in age at menarche for women born between 1920 and 1979 in Rio de Janeiro, Brazil. Ann Hum Biol. 2000;27:423-8.

5. Junqueira-do-Lago M, Faerstein E, Lopes CS, Werneck GL. Family socioeconomic background modified secular trends in age at menarche: evidence from the Pró-Saúde Study (Rio de Janeiro, Brazil). Ann Hum Biol. 2003;30:347-52.

6. Duarte MFS. Maturação física: uma revisão da literatura, com especial atenção à criança brasileira. Cad Saúde Pública. 1993;9(supl I):71-84.

7. Helm P, Münster K, Schmidt L. Recalled menarche in relation to infertility and adult weight and height. Acta Obstet Gynecol Scand. 1995;74:718-22. 
8. Okasha M, McCarron P, McEwen J, Smith GD. Age at menarche: secular trends and association with adult anthropometric measures. Ann Hum Biol. $2001 ; 28: 68-78$.

9. Sharma K, Talwar I, Sharma N. Age at menarche in relation to adult body size and physique. Ann Hum Biol. 1988; 15:43 I-4.

10. St George IM, Williams S, Silva PA. Body size and the menarche: the Dunedin Study. J Adolesc Health. 1994; 15:573-6.

I I. Crespin J. Estatura e idade à menarca e estatura definitiva. Estudo retrospectivo de 120 adolescentes. Pediatr Mod. 1990;6:403-10.

12. Gullo A. Indagini sui rapporti tra eta del menarca e statura definitiva della donna. (Contributo alla questione dell'accelerazione secolare pandemica dello sviluppo). Sicil Sanit. 1965;3:35-7.

13. Lobato DS, Silveira DD. Idade de ocorrência da menarca na zona meridional do Rio Grande do Sul e sua relação coma estatura final das mulheres. Hospital. (Rio J) 1969;75:375-8.

14. Mulligan J, Bailey BJR, Voss LD, Betts PR. Pubertal growth of the short normal girl. Horm Res. 1999;52:26I-8.

15. Hernández FG, Mendonza WL, Haddad EH. Antropometria y menarquia. An Anat Norm. 1988;6: 17। -5.
16. Shangold MM, Kelly M, Berkeley AS, Freedman KS, Groshen S. Relationship between menarcheal age and adult height. South Med J. 1989;82:443-5.

17. World Health Organization. Physical status: the use and interpretation of anthropomety. Geneva: WHO; 1995. p.397. (WHO technical report series)

18. Faerstein E, Lopes CS, Valente K, Plá MAS, Ferreira MB. Pré-testes de um questionário multidimensional autopreenchível: a experiência do estudo pró-saúde. Physis. (Rio J) 1999;9: I 17-30.

19. Eveleth PB, Tanner JM. Worldwide variation in human growth. Cambridge: Cambridge University Press; 1990.

20. Sichieri R, Taddei JA, Everhart JE. Influence of parental height and sociodemographic factors on adolescent height in Brazil. J Adolesc Health. 2000;26:4|4-9.

Artigo recebido: 25/04/05

Aceito para publicação: 27/08/06 